\title{
Application Design Framework for a Dressed-up Touchscreen
}

\section{with a Tactile Feedback.}

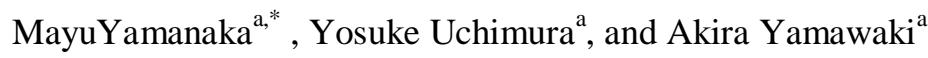 \\ ${ }^{a}$ Kyusyu Institute of Technology, 1-1 Sensui-cho Tobata-kuKitakyuusyuu-shi Fukuoka-ken, 804-8550, Japan \\ *Corresponding Author. yamanaka@boss.ecs.kyutech.ac.jp
}

\begin{abstract}
It is difficult for persons who are unfamiliar with a touchscreen like elderly or handicapped people to operate the electronic products employing touchscreen as user interface. We aim to realize a touchscreen that can provide the position of buttons and click feeling by a simple mechanism, keeping the flexibility. The proposed touchscreen consists of a switch underneath an LCD panel and the plates with identification number at the top of the LCD panel. When the touchscreen is pushed, the switch underneath the LCD module is also pressed. Thus, the user can get certain click feeling by the switch. For the plate put on the top of the LCD module, the places where icons are displayed in the touchscreen are hollowed out. Therefore, the edges around the buttons on the hollowed part give the user's finger unevenness. That is, the position and boundaries of the icons can be recognized by the finger. In addition, since the type of the plate can be determined by the ID, we can provide a variety of button arrangement according to the plate used.In order to make our touchscreen widely used, a generic design framework of software is needed. Thus, this paper shows a general framework of software, design framework, is showed.Through the application examples on the prototype, we demonstrate that the application can be developed actually byfollowing our design framework.
\end{abstract}

Keywords: touchscreen, button, framework, welfare.

\section{Introduction}

It is difficult for persons who are unfamiliar with a touchscreen like elderly or handicapped people to operate the electronic products employing touchscreen as user interface [1]. What cannot recognize the buttons on the touchscreen is one of the significant problems to make the operation more difficult.

Therefore, the systems supporting operations of a touchscreen by giving fingersatouch feeling have been developed in the past[2-7]. Their systemsare not the same as the sense of the button switch with a click feeling that we are accustomed to. In addition,the users cannot recognize button positions on their systems, and they are notsuitablefor downsizing of device.

We aim to realize a touchscreen that can provide the position of the button and the click feeling by a simple mechanism, keeping the flexibility.The flexibility means that our touchscreen is able to adapt to any user interface when changing the button sheet.Actually, we have proposed the necessary hardware framework to comprise the features described above[7].Also, we have developed a prototypedhardware according to the hardware framework. We had some testees operate the prototype. Then, it resulted that our touchscreen is easier to manipulate than the conventional one. Accordingly, the result indicates usability of our proposal. In order to make our touchscreen widely used,we need not only the general framework of the hardware shown in [7] but also a general framework of the software,design framework.According to the design framework, any programmers can develop uniformly the various applications on the touchscreen proposed.

In this paper, after we briefly explain the hardware framework of the proposed touchscreen, we describe the design framework of the application.Then, through the application examples on the prototype, we demonstratethat an application can be developed actually according to the design framework.

\section{Hardware framework}

Fig.1 shows a hardware framework of the proposed touchscreen system.Mainly, the proposed touchscreen 


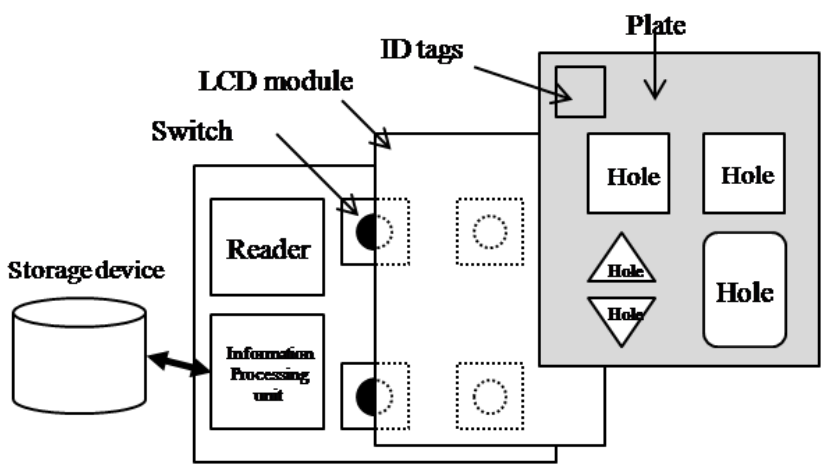

Fig.1. Hardware framework.

consists of a switch underneath the LCD panel and the plates with identification number at the top of the LCD panel.

When a finger pushes the touchscreen, the switch underneath the LCD panel is also pressed. Thus, this touchscreen gives the user the click feeling that is the same as that of mechanical switch we are accustomed to. Since the touchscreen is only executed by pushing the switch, an incorrect input by just touching can be prevented easily.

For the plate put on the top of the LCD panel, the places where icons are displayed in the touchscreen are hollowed out. Therefore, the edges around the buttons on the hollowed part give the user's finger unevenness. That is, the position and boundaries of the icons can be recognized by the finger. The ID can be read by placing the plates on the reader, the system can determine the type of plate. Therefore, the proposed touchscreen can correspond to the various arrangement of the button. In addition, the plate can be used or not can be use depending on the application. Information such as the placement of the buttons on the screen hierarchy and each layer of the plate and default is stored in a storage device. The necessary information according to the plate switching and button pushing is selected from the storage device.

\section{Design framework}

Fig.2 shows a design framework of the proposed touchscreen system.

We explain the concept called an object in each screen on the touchscreen. The object has coordinates of the button, images of icons, background images, and programs invoked by pushing a button. By the state transitions among objects invoked by pushing the buttons, the screen switching or the execution content switching is realized.

The object holds the number of the buttons $(\mathrm{m})$ and the

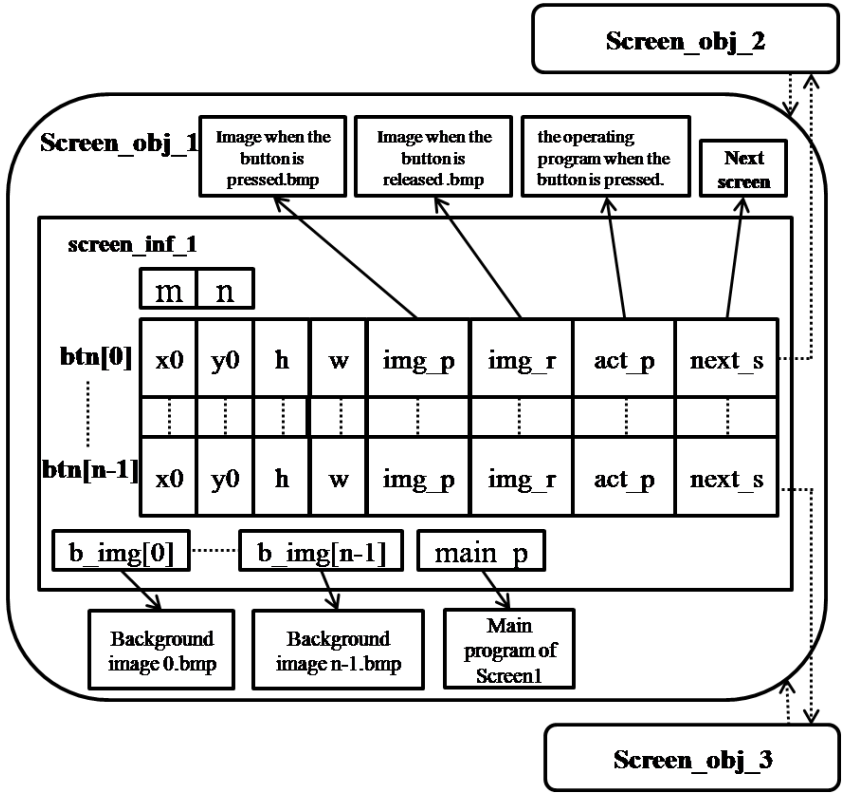

$\mathrm{x} 0, \mathrm{y} 0$ : origin coordinates of the touch button $\mathrm{h}, \mathrm{w}$ : width and height of the touch button

\section{Fig.2. Software framework.}

number of background images(n).Each button has coordinates of the origin ( $\mathrm{x} 0, \mathrm{y} 0)$, and the height and the width of the icon $(\mathrm{h}, \mathrm{w})$. Theimg_p of each button is a pointer to the image displayed when the button is pushed.The $I m g_{-} r$ is a pointer to the image displayed when the button is released. The act_pis a pointer to a program to be executed when the button is pushed. The next_s is a pointer to the next object to transition when the button is pressed.

According to the concept of Fig.2, basic function groups(library) to develop applications are provided. For example,they are the function to display the image designated for the area of the LCD, the function to be displayed to read background image from the SD card and so on.

Incidentally, it is possible to switch among multiple applications by changing the plates shown in Fig.1. A plate corresponds to one or more applications. Each application corresponds to one or more objects.

\section{Adaptation example}

We have developed an application using the proposed design framework. This case study develops a calculator on our prototype hardware. Incidentally,the application of the developed calculator can be four arithmetic operations,and is the same as the use of a common calculator. 


\subsection{System Overview}

We explain the structure of the prototype that was used to validatewhether our design framework can realize an application or not.Fig.3 shows a block diagram of the prototype.

The LCD module consists of the LCD with the $240 \times 320$ of 3.2 inch resistive type touchscreen, the LCD controller of SolomonSystechSSD1289and the AD converter for the touchscreen of SHENZHEN XPTEK TECHNOLOGY xpt2046.

This prototype usesthe plateID as RFID. The RFID reader is ID Innovations ID-12.The switches underneath the LCD module are push-button switches of OMRON B3F5000.

These three parts are connected to the microcomputer board of Strawberry Linux STBee that equipped with ST Micro's STM32 microcontroller.

Software to run on STM32calculates the X-Y axis of the finger sent from the $\mathrm{AD}$ converter attached to $\mathrm{LCD}$ module .Depending on the pressing of the switch, the microcomputer performs the processing corresponding to the default state and the current plate.

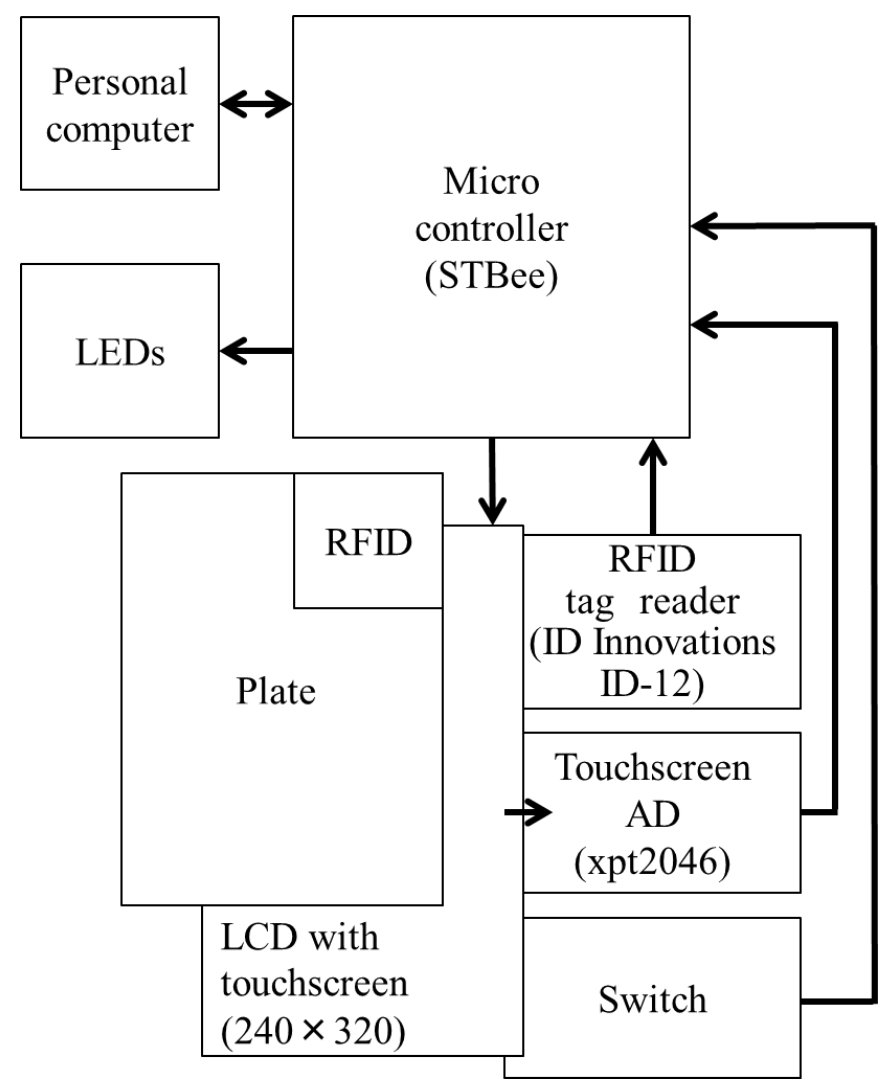

Fig.3. Block diagram.

\subsection{Application design and operation check}

Fig.4shows the appearance of the calculator we designed and the operation example.

In this case study, the plate we madecorresponds to one application and the application has one object.The number of images of the touch button (m) is 16. They include 0-9 of numbers, the sign of the four arithmetic operations, the clear, and the equal sign. The number of the background image ( $n$ ) is one.The images when pushed and released are same. When each numeric button is pushed,the numeral of the pushed button is displayed in the background image and memorized. Whena calculation button is pushed, the action performs four arithmetic operations corresponding to the kind of pushed button, by using memorized numerals. Finally, the action displays the operation result.

\begin{tabular}{|c|c|c|}
\hline & & 5 \\
\hline CA & + & $\%$ \\
\hline$x$ & $=$ & $\div$ \\
\hline$\Rightarrow$ & - & $\sqrt{ }$ \\
\hline 1 & 2 & 3 \\
\hline 4 & 5 & 6 \\
\hline 7 & 8 & 9 \\
\hline$+1-$ & 0 & $\cdot$ \\
\hline
\end{tabular}

Fig.4(a). The enlarged image of the screen.

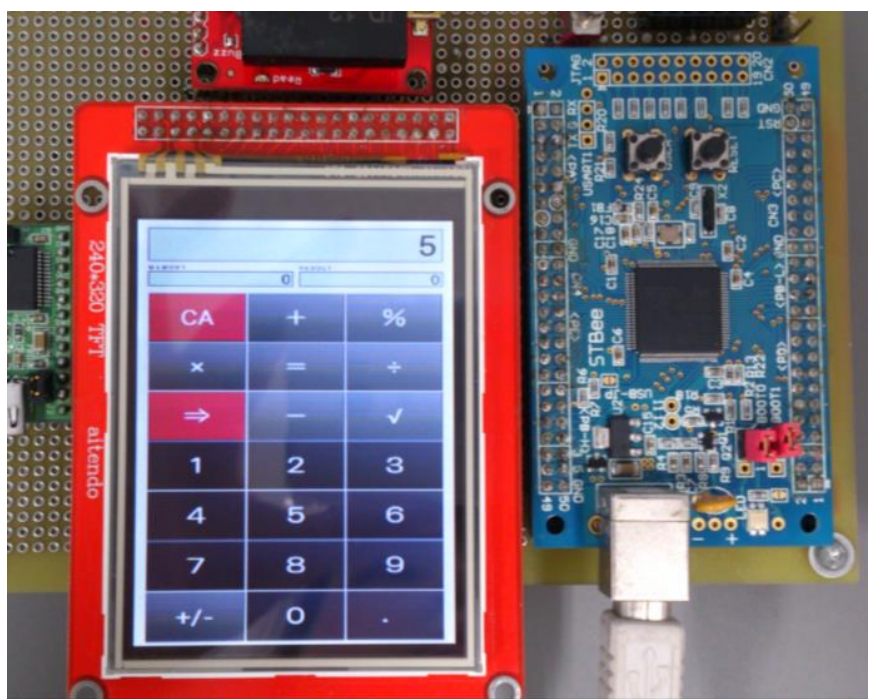

Fig.4(b). The state of the whole device.

Fig.4. Appearance of calculator and itsoperation example. 


\section{Conclusion}

In this paper, we proposed a design framework for application in the dressed-up touchscreen with a tactile feedback.Further, this paper indicates that the application can be developed in practice by using the design framework through a case study of a calculator.

As future work, we will increase application examples, and perform an experiment to the more testees.

\section{References}

[1]Masatomo Kobayashi, Atsushi Hiyama, Takahiro Miura, Chieko Asakawa, Michitaka Hirose, and TohruIfukube: "Elderly User Evaluation of Mobile Touchscreen Interactions", Human-Computer Interaction - INTERACT 2011, Lecture Notes in Computer Science, Vol.6946, pp.83-99, 2011.

[2]David McGookin, Stephen Brewster, and WeiWei Jiang: "Investigating Touchscreen Accessibility for People withVisualImpairments",Proceedings of the 5th Nordic conference on Human-computer interaction, pp. 298-307, 2008 .

[3] Koji Yatani and KhaiNTruong: "SemFeel: A User Interface with Semantic Tactile Feedback for Mobile Touch-screen Devices", Proceedings of the 22nd annual ACM symposium on User interface software and technology, pp. 111-120, 2009.

[4]Olivier Bau, Ivan Poupyrev, Ali Israr, and Chris Harrison, "TeslaTouch: Electrovibration for Touch Surfaces", Proceedings of the 23rd annual ACM symposium on User interface software and technology, pp.283-292, 2010.

[5]Masahiko Kawakami, MasaruMamiya, TomonoriNishiki, Yoshitaka Tsuji, Akito Okamoto, and ToshihiroFujita: "A New Concept Touch-Sensitive Display Enabling Vibro-Tactile Feedback", Proceedings of HCI on People and Computers XIII, pp.303-312, 1998.

[6] Tactus Technology: "Tactile user interface for touch-screen devices", http://www.tactustechnology.com/, 2012.
[7]Mayu Yamanaka, RyohsukeSuzue, and Akira Yamawaki:"The realization of the dressed-up touchscreen with a tactile feedback",Proceedings of the 1st International Conference on Industrial Applications Engineering 2013,pp.21-27, 2013 